\title{
All that glitters is gold-frozen fish
}

For Mary Hagedorn, it was a moment over twenty years in the making: stirring before her was a zebrafish embryo, alive and seemingly well after taking a dip to $-196{ }^{\circ} \mathrm{C}$. Hagedorn is a conservation biologist at the Smithsonian Conservation Biology Institute and Hawaii Institute of Marine Biology with a long interest in cryopreservation. "I've cryopreserved easily over a million, maybe even more, zebrafish embryos in my life and every single one of them turned to mush," she recalls. "To see one even intact for fifteen minutes was a huge, huge thing. And then to see them move at 24 hours was mind-blowing." The trick was a laser and a little gold (ACS Nano 2017; doi:10.1021/acsnano.7b02216).

Cryopreservation of living cells first left the realm of science fiction in the 1950s. The basic approach involves freezing and thawing a sample quickly and uniformly to prevent the formation of ice crystals. Adding cryoprotectants helps prevent ice damage and smooths the "vitrification" process by which the sample essentially turns to glass. Over the decades, cryopreservationists became pretty adept at handling mammalian cells and embryos, including those of humans. But fish, and other nonmammalian organisms like reptiles, amphibians, and birds, remained out of reach.

Hagedorn joined the Smithsonian in the 1990 s to explore why. With biophysicist Fritz Kleinhans at IUPUI, she developed a biophysical model to better understand the cryopreservation challenges inherent to zebrafish, which was then a rapidly emerging model organism.

The underlying issues were two-fold. Zebrafish embryos are physically largethey need to be self-sustaining, so they include a large yolk to supply nutrientsand their internal membranes are quite impermeable to water to prevent bursting from changes in osmotic pressure. Ice formation is problematic with larger volumes,
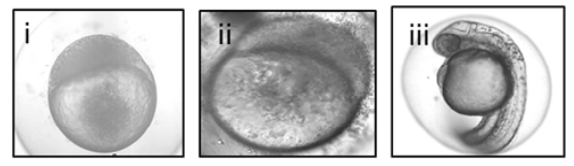

A cryopreserved embryo at (i) 1 , (ii) 3, and (iii) 24 hours after laser-rewarming. Adapted with permission from ACS Nano 2017;

doi:10.1021/acsnano.7b02216. Copyright 2017 American Chemical Society

and cryoprotectants do not diffuse into the embryo's different compartments as easily as in other species. To overcome those issues, Hagedorn and her collaborators took advantage of microinjection technology to improve cryoprotectant delivery. This advance enabled them to deep-freeze countless embryos.

But they couldn't bring them back. Technology at the time was just not capable of warming them fast enough to prevent ice damage, Hagedorn says, so in 2004 she stopped...until a cryopreservation conference nearly a decade later. John Bischof, a mechanical engineer at the University of Minnesota, was presenting his lab's work with an emerging approach called laser warming. A new collaboration between biology and engineering began, led by Bischof's PhD student Kanav Kholsa.

Unlike convective methods, laser warming relies on an absorber that indirectly transfers heat to surrounding tissue. Kleinhans and Peter Mazur at IUPUI had successfully laser-warmed mouse oocytes surrounded by India ink, but zebrafish embryos were still too large (and internally complex) for an external absorber to be sufficient. No matter-Hagedorn had worked out that issue in her earlier work with cryoprotectants. They just needed a biocompatible absorber that could be microinjected into the zebrafish embryo along with the cryoprotectant. Their solution was golden-literally.

Gold, Kholsa explains, is inert and has long been used in biomedical applications.
It also absorbs light in the wavelength of the Bischof lab's laser: 1,064 nm. Kholsa devised a mixture of the cryoprotectant polyethylene glycol and gold nanoparticles, microinjected it into 223 embryos, and froze them with liquid nitrogen. Then he hit a frozen embryo with the laser, a step lasting just one one-thousandth of a second.

Though 223 fish didn't bounce back to life and normal development, a handful did, a feat that had eluded researchers for decades. From this proof-of-concept, Kholsa is ready to refine. "I would say this first paper, it was a lot of scaling, a lot of calculations and modeling and lot of trials to figure out that this can be done," he says. "Right now, we are just showing that there's structure and some functionality after the re-warming." In the future, he hopes to determine what exactly is happening to the embryo in the incredibly short warming burst, and to improve the nanoparticles and their distribution throughout a sample.

In addition to zebrafish, the team thinks that laser warming could be extended to many other organisms that have been beyond the reach of cryopreservation in the past. That has important value to Hagedorn, who currently studies coral conservation. "It's transformational in what it will allow us to do to conserve species," she says, "Because really all you have to do is freeze them. The laser function can happen later." Theoretically, says Kholsa, the technique could be applied to any 1-2 mm embryo or tissue sample; the nanoparticles used and temperature targets can be adjusted as needed.

Hagedorn looks forward to deploying the technique at her lab in Hawaii, where Kholsa will join her for a few weeks to think through how to increase throughput and mechanize the thawing process. "Engineers can do amazing things," she says, "and we're just going to keep plucking away at it.”

Ellen P. Neff 